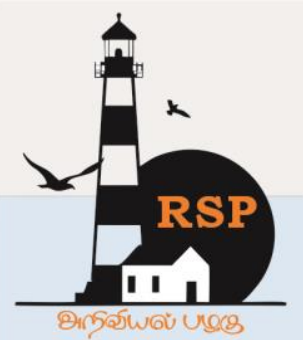

INTERNATIONAL RESEARCH JOURNAL ON

e-ISSN : 2582 - 4376 ADVANCED SCIENCE HUB Open Access

RSP SCIENCE HUB

(The Hub of Research Ideas)

Available online at www.rspsciencehub.com

\title{
Fisherman Detecting System Using IOT
}

Arul Murugan $V^{1}$, Jeevitha $G^{2}$, Jothikrishnan $M^{3}$, Nandha Kumar $S^{4}$, Pooja $S^{5}$

${ }^{1}$ Professor, Department of Electrical and Electronics Engineering, Excel College of Engineering and Technology, Namakkal, Tamil Nadu, India

2345 Student, Department of Electronics and Communication Engineering, Excel College of Engineering and Technology, Namakkal, Tamil Nadu, India

Abstract

In a Tamilnadu, each and every second, our one of fisherman life have risk when who are surveying their life in sea. Mostly Sri Lankan solders are treaded our fisherman as prison and take custody and sometime their life is gone. All these kind of issue occur because identifying sea border between countries. Our paper will provide solution to this problem and improve safety of fisherman life in all point of view. For this, we have designed an embedded system to notifying sea border of our country by using IOT (Internet Of Things) and RF Transmitter Receiver. The embedded unit control overall system performance and compare pre-defined and present value of fisherman position and inform to them about their current location whether they are in safety zone or restricted zone. IOT and RF communication units' provider location of all zones of sea. The embedded unit interface with fisherman by LCD display. The paper aims at providing a system that will alert the fishermen well in advance and ensure maximum safety and peace at the border and also notify the family members and patrol system using by software. This will help and increase safety of fisherman life.

Keywords: Fisherman, IoT, Embedded control unit

\section{Introduction}

Recently unmanned border patrol system consisting of high tech devices, like unmanned aerial vehicles, surveillance towers equipped with wireless camera used to monitor fishermen whose family's main economical support is fishing. From Tamil Nadu about 18,000 boats of different kinds conduct fishing along the India-Sri Lanka border. But accidentally crossing border without knowledge, they get shot by the Lankan navy. This leads to loss in the both humans as well as their economic incomes. We have developed a system which eliminates such problems and saves the lives of the fishermen. Border systems have recently achieved interest to address concerns about national security. The major problem in protecting long stretches of borders is the need for large human involvement. This system is designed to avoid such kind of accidents and to alert the fishermen about border area well before using latest technology of Global Positioning System (GPS) and Global System for Mobile communication (GSM) and monitored using IOT.

\section{Literature Survey}

Literature survey earlier to begin a research project is essential in understanding fishermen order alert system, as essential this will supply the researcher with much needed additional data on the methodologies and technologies available and used by other research complement around the world. This chapter provides a compressed summary of literature reviews on key topics which related to fishermen border alert systems. 


\subsection{Fishermen Nautical Border Alert System:}

In day-to- day life we hear about many Tamil fishermen being caught and put under Sri Lankan custody and even killed. The sea border between the countries is not easily identifiable, which is the main reason for this cross border cruelty. We use GPS receiver to find the current location of the fishing boat or vessel. Using GPS, we can find the current latitude and longitude values and is sent to the microcontroller unit. Then the controller unit finds the current location by comparing the present latitude and longitudinal values with the predefined value. Then from the result of the comparison, this system aware the fishermen that they are about to reach the nautical border. If the boat is in normal area, then the LCD displays normal zone. Thus they can make it clear that the boat is in normal area. In case it moves further and reaches the warning zone, the LCD displays warning zone. [1-4] If the fisherman ignores the warning or fail to see the display and move further and if the boat enters the zone nearer to the restricted zone the alarm will turn on and the speed of the boat engine automatically gets controlled by $50 \%$. If the fisherman did not take any reaction about the alarm and move further, then the boat will enter into the restricted zone, the alarm continues to beep as before, and once it touches the restricted zone, the boat engine gets off by the control of fuel supply to engine.

\subsection{Implementation of Maritime Border Alert System:}

This paper proposed to help the small scale fishermen for safe navigation in maritime and then preventing them from entering other country border line. Data collection unit consists of GPS thus provided the information of location based on the position the boat and transmitter. The processing unit fetched with already known details of border between the countries, and comparison being done with known data and current position data. The controlling unit will make decision in order to alert the fishermen and coat guards.

\subsection{Passive RFID Tag based Heart Rate Monitoring from an ECG Signal:}

Passive RFID (Radio Frequency Identification) tags work on wireless power harvested from a RFID reader and thus do not require batteries for operation. RFID tags have been conventionally used as product identification tools similar to barcodes. For these tags to be used in sensor networks, they have to be capable of transmitting sensor data along with the tag ID. In its simplest form, an RFID tag can be used as a one bit transmission device by turning the RFID tag on/off and having an RFID reader detect the tag's state. For instance, the reader detects a ' 1 '. When the tag is on and a ' 0 ' when the tag is off. A similar device that uses two RFID tags . Contemporary RFID tags are capable of transmitting multiple bits of optional data along with the default tag ID.[5-8] However, additional circuitry like analog to digital converters (ADCs) and microcontrollers are required to digitize and embed the sensor data with the tag ID. These additional components not only increase the size of the system but also add to its power requirements. Another drawback of such systems is that they require significant transmitted data redundancy to achieve a degree of reliability. For example, the electroencephalogram (EEG) system proposed in requires $92 \%$ data overhead.[9-13]

\subsection{Alert System for Fishermen Border crossing using Android:}

The application can be widely used by people in the border to find the appropriate path to reach the destination. The notification will be sent to the border security forces which act as the server to all other devices that are operated by people in ships. The application will notify the information of where the devices are being located and intimate them about the issues that occur due to opponent forces in ships to server. This is processed mainly for Tamil fishermen's who are involved in fishing. The application uses the Global Positioning System (GPS) to provide the latitude and longitude information and its being used for tracking devices. The system entirely uses the device based tracking which avoids failure in the system due to network problems. The tracking here totally depends on the device and not on the signal/network that is currently used.

\subsection{Location based Services using Android:}

Initially mobile phones were developed only for voice communication but now days the scenario has changed, voice communication is just one aspect of a mobile phone. There are other aspects which are major focus of interest. Two such major factors are web browser and GPS services. Both of these functionalities are already implemented but are only in the hands of manufacturers not in the hands of users because of proprietary issues, the system does not allow the user to access the mobile 


\section{www.rspsciencehub.com}

hardware directly. But now, after the release of android based open source mobile phone a user can access the hardware directly and design customized native applications to develop Web and GPS enabled services and can program the other hardware components like camera etc. In this paper we will discuss the facilities Available in android platform for implementing LBS services (geoservices).

\subsection{RFID and GPS Combination approach Implementation in Fisher Boat Tracking System:}

Wireless sensor networks have potential to provide unprecedented remote monitoring. capabilities that can benefit applications such as industrial control, environmental control, and defence. Radio Frequency Identification (RFID) is one of the most exciting technologies that revolutionize the working practice by increasing efficiencies, and improving profitability. It is also presented as a converting of today's barcodes, but the innovative technology has much largest Possibilities such as individual serial numbers for each items, and the possibility to read these numbers at a distance of several meters. They are simpler to deploy than wired salutations and these networks will enabled improved understanding of processes and environments through continual monitoring of a larger set of parameters. In this project evolution of wireless sensor networks will be presented, using RFID.

\section{Existing System}

The existing system using a GPS receiver which receives signal from the satellite and give the current location position of the boat. The existing system is used to detect the border of the country through the specified longitude and latitude of the position, not only between Sri Lanka and India but all over the world. The particular layer level i.e. border can be predefined and this can be stored in microcontroller memory. The current value is compared with predefined values and if these values are same, immediately the particular operation will be done i.e., the microcontroller gives instruction to the alarm to buzzer. It also uses a message transmitter to send message to the base station which monitors the boats in the sea. The system provides an indication to both fisherman and to coastal guard.

\subsection{Block Diagram Decription:}

As the name suggests, main aim of the system is to prevent fishermen's from crossing boundaries. Microcontroller 89s52 acts as a main control block of the proposed system. GPS modem SR 67 is interfaced to the system using UART protocol. Modem continuously transmits the data containing location and other information. This data is read by the microcontroller which fetches the location information. This information is in the standard NMEA protocol data format. GPS modem transmits the data in RS232 protocol, whereas microcontroller supports TTL, hence MAX 232 acts as a protocol convertor. GSM SIM900 modem is used to add SMS alert facility to the system. Microcontroller is pre-programmed with boundary location for that particular geographical area. It continuously reads data from the GPS and compares with the boundary location; if the current location to be found is nearer to the boundary the microcontroller alerts the user using a buzzer.

If system tends to get more and more closer to the border, an SMS is sent to the control room for alert, simultaneously ringing the alarm. In case boat reaches the border and the location matches the pre-stored boundary the engine is made automatically off by the system. LCD acts as a user interface which continuously displays Latitude and Longitude co-ordinates. $12 \mathrm{~V} 1.3$ AH Battery is used to power up the system. LM 7805 used as a voltage regulator IC which provides $+5 \mathrm{~V}$ constant DC supply. Relays used to control the engine operation. ULN 2803 acts world.

\subsection{Disadvantages:}

- Accurate position information becomes critical.

- Cost is high.

- Lack of stopping boat system.

- Less time efficiency.

\section{Proposed Work}

In the proposed method we overcome the disadvantages present inside the existing method by developing microcontroller and RFID tag. Also developed the VB software used to enroll the fishermen's data. In the proposed system a solution is given to the problem of "Fishermen Tracking their location in the sea" for this the sea area is divided into three zones namely safe, intermediate and danger. This system will provide communication with customer like fisherman family and control 
room by using RFID tag. This will help and increase safety of the fisherman life.

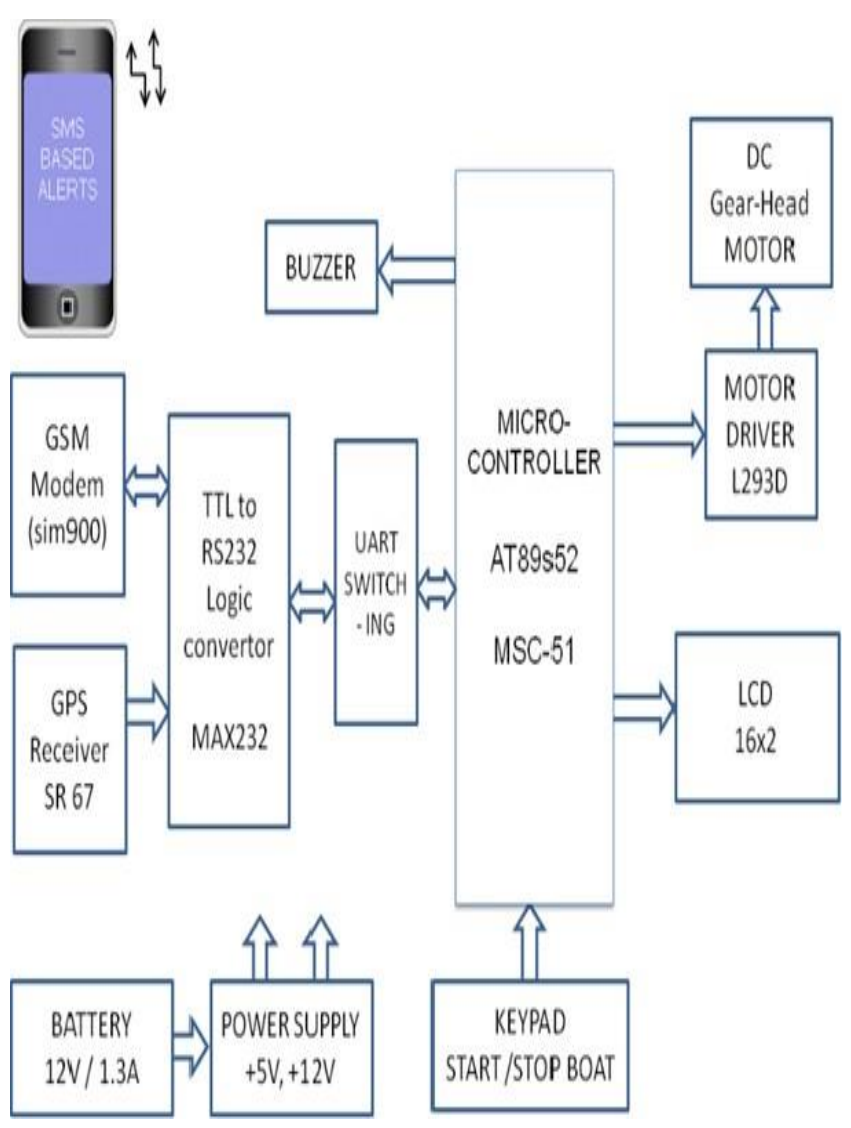

Fig .1 Block diagram of existing system

\subsection{Block Diagram Description:}

The Proposed system has the followingunits,

1) Embedded or controlunit.

2) Communication unit.

\section{1) RF transmitter}

\section{2) $\mathrm{RF}$ receiver}

3) Indication unit.

\section{Hardware Requirements}

\subsection{Embedded or Control Unit}

The controller unit consists of the following unit,

a) Power supply

b) Microcontroller ATmega

c) DC motor

d) Relay

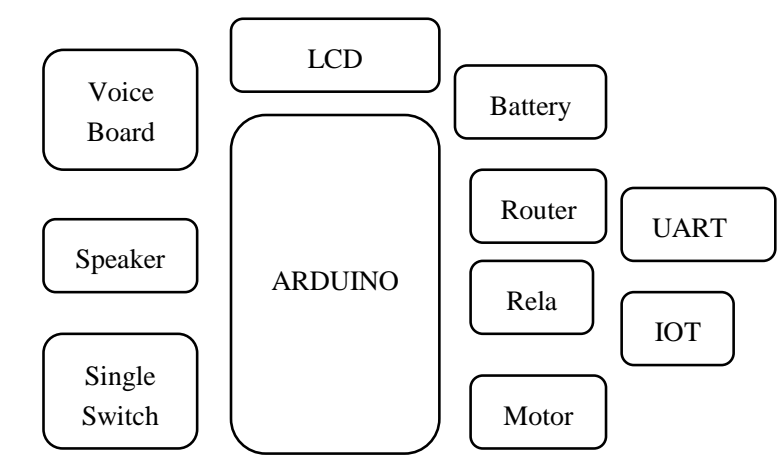

Fig.2 Block diagram of Proposed system

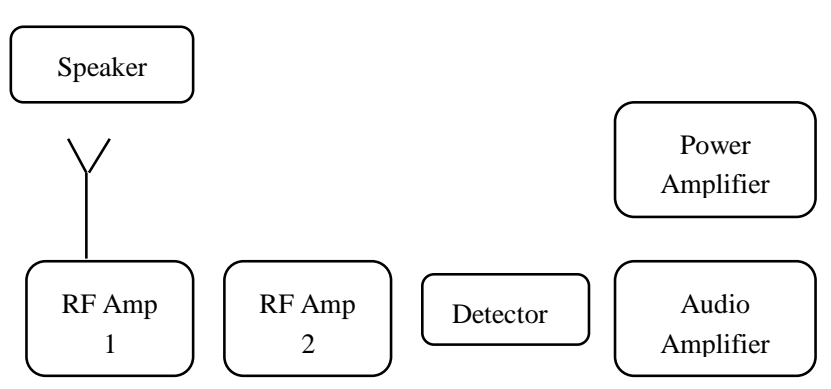

Fig. 3 RF Receiver

Audio Signal
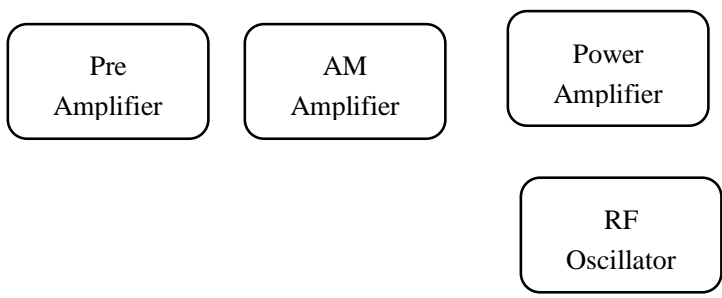

Fig. 4 RF Transmitter

\section{1 (a) Power Supply:}

Available power source is an Ac voltage arrives at 230V.Since our electronic circuits require only very minimal voltage and current we use step down power transformer. Step down transformer is designed in such a way that the input is $230 \mathrm{~V}$ and output of $12 \mathrm{~V}$. Another thing is that electronic circuits operate in DC whereas available output of transformer is Ac of $12 \mathrm{~V}$. So rectifier circuit is used to convert AC to DC. Rectifier circuit consists of four diodes formed in bridge fashion so as to convert incoming AC to DC.

\section{Block Diagram:}




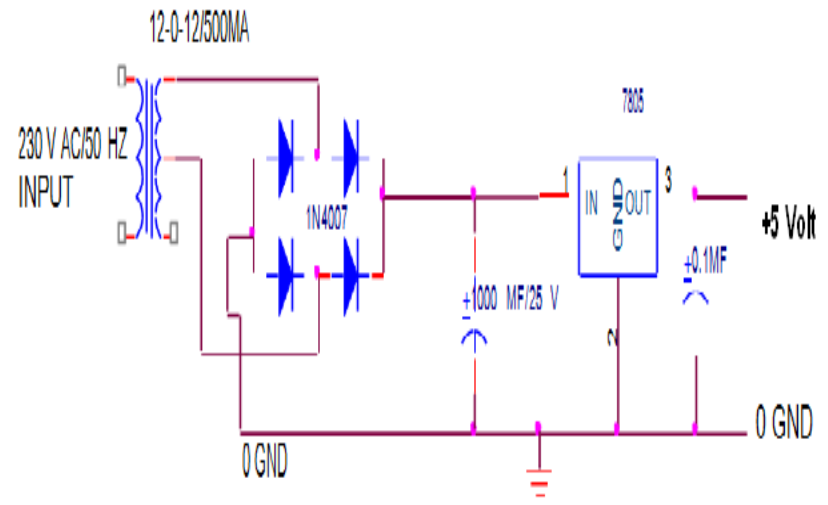

Fig. 5 Power Supply Circuit Diagram

\section{1(b) Arduino Microcontroller (Atmega328P):}

Arduino/ Genuino is a microcontroller board based on the Atmega328P (datasheet). It has 14 digital input/output pins (of which 6 can be used as PWM outputs), 6 analog inputs, a $16 \mathrm{MHz}$ quartz crystal, a USB connection, a power jack, an ICSP header and a reset button. It contains everything needed to support the microcontroller; simply connect it to a computer with a USB cable or power it with an AC-to-DC adapter or battery to get started. You can tinker with your UNO without worrying too much about doing something wrong, worst case scenario you can replace the chip for a few dollars and start over again.

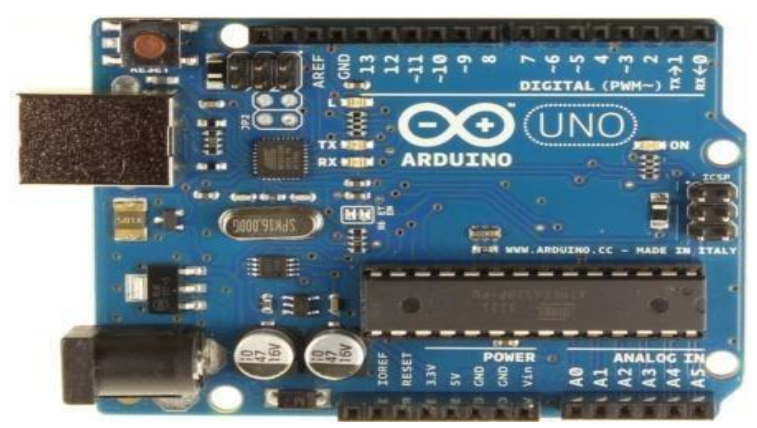

Fig. 6 Arduino Uno R3 microcontroller

Arduino is a computer hardware and software company, project, and user community that designs and manufactures microcontroller kits for building digital devices and interactive objects that can sense and control objects in the physical world. The project's products are distributed open source hardware and software. Which are licensed under the GNU Lesser General Public License (LGPL) or the GNU General Public License (GPL), permitting the manufacture of Arduino boards and software distribution by anyone. Arduino boards are available commercially in preassembled form, or as do-it-yourself kits. Arduino board designs use a variety of microprocessors and controllers. The boards are equipped with sets of digital and analog input/output $(\mathrm{I} / \mathrm{O})$ pins that may be interfaced to various expansion boards (shields) and other circuits. The boards feature serial communications interfaces, including Universal Serial Bus (USB) on some models, which are also used for loading programs from personal computers. The microcontrollers are typically programmed using a

dialect of features from the programming languages $\mathrm{C}$ and $\mathrm{C}++$. In addition to using traditional compiler tool chains, the Arduino project provides an integrated development environment (IDE) based on the Processing language project. Many Arduino-compatible and Arduino derived boards exist. Some are functionally equivalent to an Arduino and can be used interchangeably. Many enhance the basic Arduino by adding output drivers, often for use in school-level education, to simplify making buggies and small robots. Others are electrically equivalent but change the form factor.

\section{1 (c) DC Motor:}

\section{Principle of Operation}

In any electric motor, operation is based on simple electromagnetism. A current-carrying conductor generates a magnetic field; when this is then placed in an external magnetic field, it will experience a force proportional to the current in the conductor, and to the strength of the external magnetic field. As you are well aware of from playing with magnets as a kid, opposite (North and South) polarities attract, while like polarities (North and North, South and South) repel. The internal configuration of a DC motor is designed to harness the magnetic interaction between a current carrying conductor and an external magnetic field to generate rotational motion.

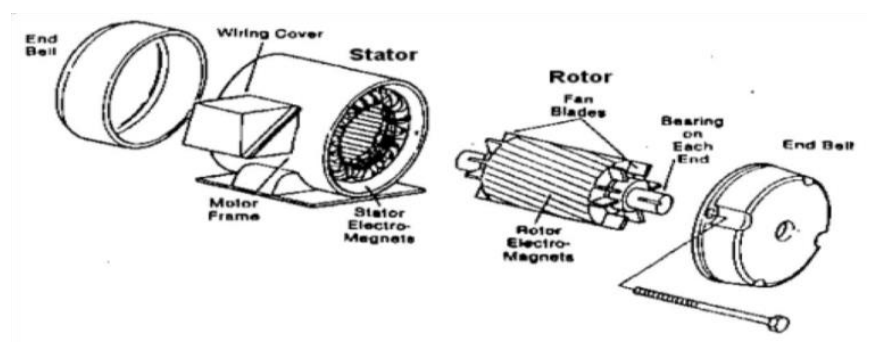

Fig. 7 DC motor 


\section{www.rspsciencehub.com}

Let's start by looking at a simple 2-pole DC electric motor (here red represents a magnet or winding with a "North" polarization, while green represents a magnet or winding with a "South" polarization). Every DC motor has six basic parts axle, rotor (a.k.a., armature), stator, commentator, field magnet(s), and brushes. In most common DC motors (and all that Beamers will see), the external magnetic field is produced by high- strength permanent magnets 1 . The stator is the stationary part of the motor - this includes the motor casing, as well as two or more permanent magnet pole pieces. The rotors (together with the axle and attached commentator) rotate with respect to the stator. The rotor consists of windings (generally on a core), the windings being electrically connected to the commentator. The above diagram shows a common motor layout - with the rotor inside the stator (field) magnets. D.C. Motor is a machine which converts electrical energy into mechanical energy. Its location is based on the principal that when a current carrying conductor is placed in the magnetic field, it experiences a mechanical force whose direction is given by Fleming's left hand rule.

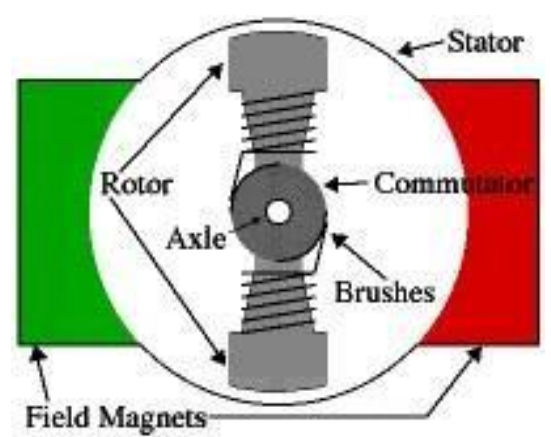

Fig. 8 Construction of DC motor

When its field magnets are excited \& its armature conductors are supplied with current from the supply mains, they experience a force tending to rotate the armature. Armature conductors under $\mathrm{N}$-pole are assumed to carry current downwards (plus) \& those under S-pole are assumed to carry current upwards (minus). By applying Fleming's left - hand rule, the direction of the force on each conductor can be found. It is shown by small arrow placed above each conductor. It will be such that each conductor experiences a force which tends to rotate the armature in anti-clockwise direction. These forces collectively produce a driving torque which sets the armature rotating.

\section{1(d) RELAY}

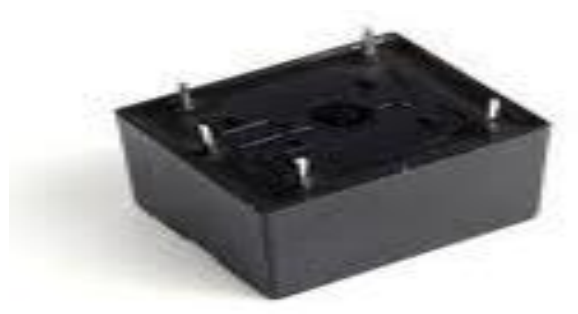

Fig. 9 Relay diagram

A relay is an electrically operated switch. Many relays use an electromagnet to operate a switching mechanism mechanically, but other operating principles are also used. Relays are used where it is necessary to control a circuit by a low- power signal (with complete electrical isolation between control and controlled circuits), or where several circuits must be controlled by one signal. The first relays were used in long distance telegraph circuits, repeating the signal coming in from one circuit and retransmitting it to another.

A relay will switch one or more poles, each of whose contacts can be thrown by energizing the coil in one of three ways:

Normally-open (NO): These contacts connect the circuit when the relay is activated; the circuit is disconnected when the relay is inactive. It is also called a Form A contact or "make" contact. NO contacts can also be distinguished as "early-make" or NOEM, which means that the contacts will close before the button or switch is fullyengaged.

Normally-closed (NC): These contacts disconnect the circuit when the relay is activated; the circuit is connected when the relay is inactive.

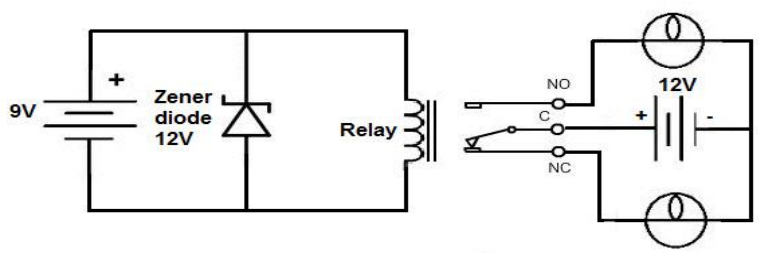

Fig. 10.DC Relay driver circuit

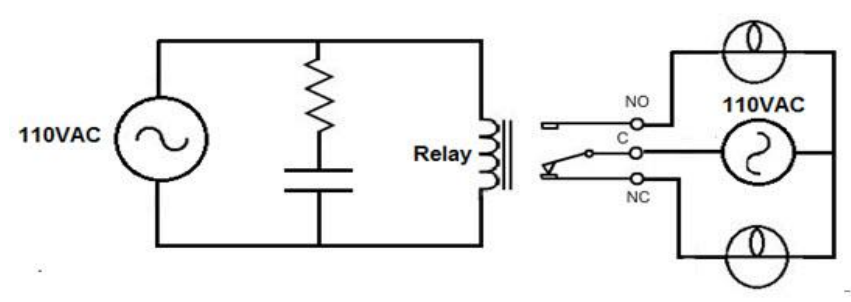

Fig. 11 AC Relay Driver circuit 
www.rspsciencehub.com

Form B contact or "break" contact. NC contacts can also be distinguished as "late- break" or NCLB, which means that the contacts will stay, closed until the button or switch is fullydisengaged.

Change-over (CO): These contacts control two circuits: one normally-open contact and one normally-closed contact with a common terminal. It is also called a Form C contact or "transfer" contact ("break before make"). If this type of contact utilizes "make before break" functionality, then it is called a Form D contact.

SPDT: Single Pole Double Throw. A common terminal connects to either of two others. Including two for the coil, such a relay has five terminals in total.

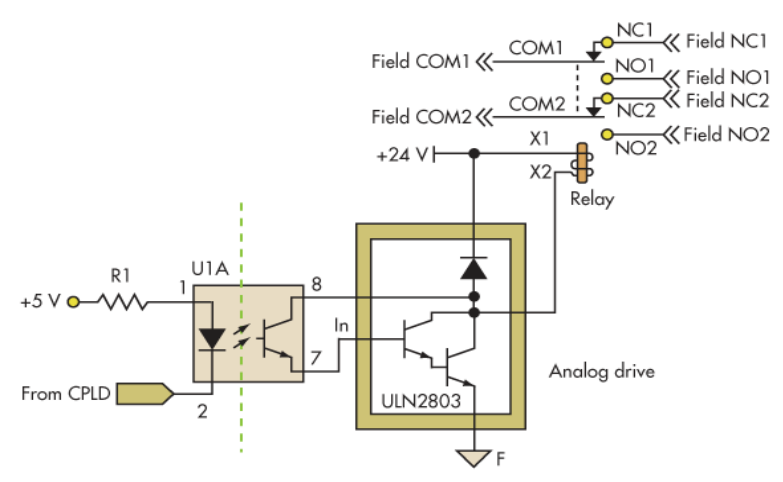

\section{Fig.12 Circuit Options Explore Issues, Solutions For Relay Drivers}

\subsection{Communication}

A communication device is a hardware device capable of transmitting an analog or digital signal over the telephone, other communication wire, or wirelessly. The best example of a communication device is a computer modem, which is capable of sending and receiving a signal to allow computers to talk to other computers over the telephone. Other Examples of communication devices include a network interface card(NIC), Wi-Fi devices, and an accesspoint.The communication unit consists of following units,
a) RF transmitter
b) RF Reciver
c) Iot
d) $\mathrm{Gps}$
e) Voice control unit

\section{2 (a) RF Transmitter:}

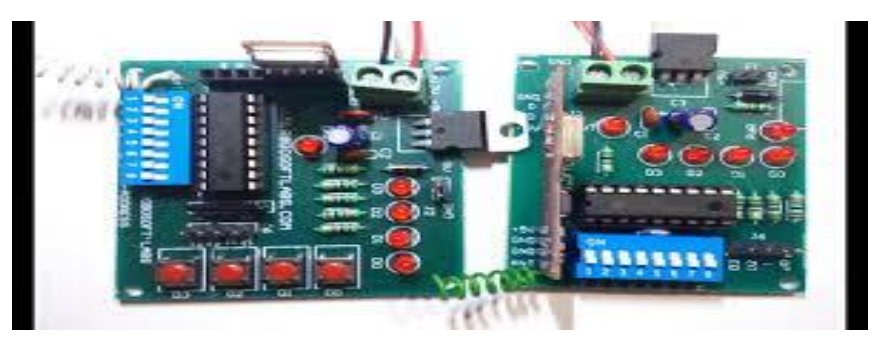

Fig. 12 Rf transmitter and reciver module

In electronics and telecommunications a transmitter or radio transmitter is an electronic device which produces radio waves with an antenna. The transmitter itself generates a radio frequency alternation current, which is applied to the antenna. When excited by this alternating current, the antenna radiates radio waves. Transmitters are necessary component parts of all electronic devices that communicate by radio, such as radio and television broadcasting stations, cell phones, walkie-talkies, wireless computer networks, Bluetooth enabled devices, garage door openers, two-way radios in aircraft, ships, spacecraft, radar sets and navigational beacons. The term transmitter is usually limited to equipment that generates radio waves for communication purposes; or radiolocation, such as radar and navigational transmitters. Generators of radio waves for heating or industrial purposes, such as microwave ovens or diathermy equipment, are not usually called transmitters, even though they often have similar circuits. The term is popularly used more specifically to refer to a broadcast transmitter, a transmitter used in broadcasting, as in FM radio transmitter or television transmitter. This usage typically includes both the transmitter proper, the antenna, and often the building it is housed in.

\section{2 (b) RF Receiver:}

In radio communications, a radio receiver, also known as a receiver, wireless or simply radio is an electronic device that receives radio waves and converts the information carried by them to a usable form. It is used with an antenna. The antenna intercepts radio waves (electromagnetic waves) and converts them to tiny alternating currents which are applied to the receiver, and the receiver extracts the desired information. The receiver uses electronic filters to separate the 


\section{www.rspsciencehub.com}

desired radio frequency signal from all the other signals picked up by the antenna, an electronic amplifier to increase the power of the signal for further processing, and finally recovers the desired information through demodulation.

Radio receivers are essential components of all systems that use radio. The information produced by the receiver may be in the form of sound, moving images (television), or digital data. A radio receiver may be a separate piece of electronic equipment, or an electronic circuit within another device. The most familiar type of radio receiver for most people is a broadcast radio receiver, which reproduces sound transmitted by radio broadcasting stations, historically the first mass-market radio application. A broadcast receiver is commonly called a "radio". However radio receivers are very widely used in other areas of modern technology, in televisions, cell phones, wireless modems and other components of communications, remote control, and wireless networking systems.

\section{2 (c) IoT:}

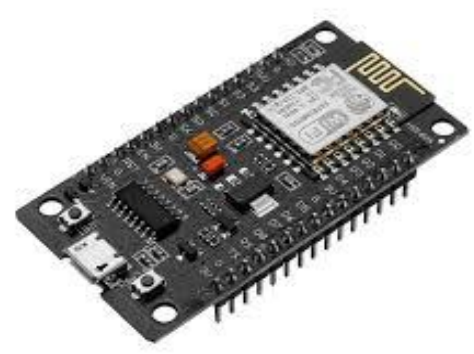

Fig. 13 IOT Module

It is used to collect and transfer data over a network without manual intervention. The embedded technology in the object helps them to interact with internal states and the external environment, which in turn helps in decisions making process.

In a nutshell, IoT is a concept that connects all the devices to the internet and let them communicate with each other over the internet. IoT is a giant network of connected devices - all of which gather and share data about how they are used and the environments in The 'Thing' in IoT can be any device with any kind of built-in-sensors with the ability to which they are operated.
Volume 02 Issue 04 April 2020

A developer submits the application with a document containing the standards, logic, errors \& exceptions handled by him to the tester. Again, if there are any issues Tester communicates it back to the Developer. It takes multiple iterations \& in this manner a smart application is created.

Similarly, a room temperature sensor gathers the data and send it across the network, which is then used by multiple device sensors to adjust their temperatures accordingly. For example, refrigerator's sensor can gather the data regarding the outside temperature and accordingly adjust the refrigerator's temperature. Similarly, your air conditioners can also adjust its temperature accordingly. This is how devices can interact, contribute \& collaborate.

\section{2 (d) GPS:}

A GPS navigation device is any device that receives Global Positioning System (GPS) signals for the purpose of determining the device's current location on Earth. GPS devices provide latitude and longitude information, and some may also calculate altitude. GPS devices are used in military, aviation, marine and consumer product applications. The accuracy of GPS depends on the type of receiver. Most hand-held GPS units have about 10-20 meter accuracy. Other types of receivers use a method called Differential GPS (DGPS) to obtain much higher accuracy. DGPS requires an additional receiver fixed at a known location nearby. Observations made by the stationary receiver are used to correct positions recorded by the roving units, producing an accuracy greater than $1 \mathrm{~m}$

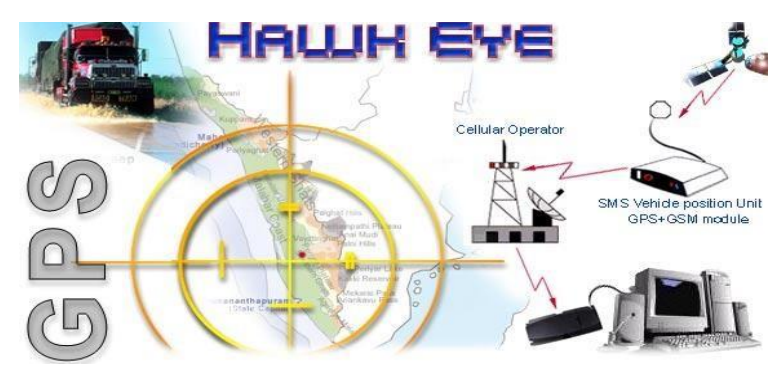

Fig.14 GPS

GPS is used in vehicles for both tracking and navigation. Tracking systems enable a base station to keep track of the vehicles without the intervention of the driver where, as navigation 


\section{www.rspsciencehub.com}

system helps the driver to reach the destination. Whether navigation system or tracking system, the architecture is more or less similar. The navigation system will have convenient, usually a graphic, display for the driver which is not needed for a tracking system. Vehicle Tracking Systems combine a number of well-developed technologies. Irrespective of the technology being used, VTS consist of three subsystems: a) In-vehicle unit (IVU), b) Base station and c) Communication link. The IVU includes a suitable position sensor and an intelligent controller together with an appropriate interface to the communication link. Thanks to the US Government announcement of $911 \mathrm{E}$ regulation, radio based position technology has witnessed a spurt of developmental activities.

\subsection{Indication}

To monitor and control an efficient floe system a full range of accurate valve automation solutions. Our automation systems provide reliable status feedback at all times, and are easy to both setup and operate. Our indication unit provides basic information about the position of valves used in hygienic application based on proven technologist's range of valve position indicators offers safe,reliable.

The following indication units are,
a) LCD Display
b) Speaker

\section{3 (a) LCD Display:}

LCD stands for liquid crystal; this is a output device with a limited viewing angle. The choice of LCD as an output device was Because of its cost of use and is better with alphabets when compared with a 7-segment LED display.

There are many kinds of LCD today and our application requires a LCD with 2 lines and 16 characters per line, this gets data from the microcontroller and displays the same. It has 8 data lines, 3 control line, a supply voltage VCC $(+5 \mathrm{v}$ and a GND. This makes the whole device user friendly by showing the balance left in the card. This also shoes the card that is currently being used.

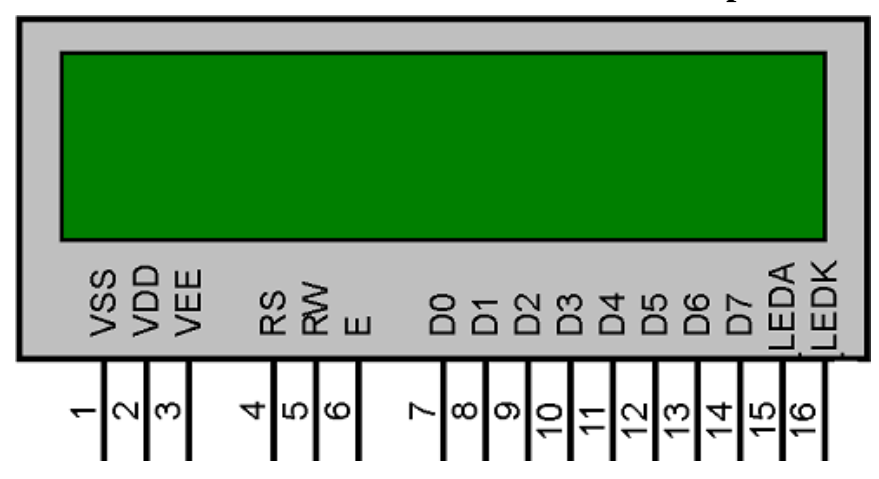

Fig. $1516 x 2$ LCD display

Fig. 15 shows the $16 \times 2$ LCD. In recent years the LCD is finding widespread use replacing LED's. This is due to the following reasons:

- The declining prices of LCD's.

- The ability to display numbers, characters and graphics.

- This is in contrast to LED's, which are limited to numbers and fewcharacters.

Incorporation of a refreshing controller into the LCD, there by relieving the CPU of the task of refreshing the LCD .in contrast, the Led must be refreshed by the CPU to keep displaying the data. Ease of programming for characters and graphics

\section{3 (a) (i) VCC, VSS and VEE}

While VCC and VSS provide $+5 \mathrm{v}$ and ground respectively, VEE is used for controlling LCD contrast.

\section{3 (a) (ii) RS, Register Select}

There are two very important registers inside the LCD. The RS pin used for their selection as follows. If $\mathrm{RS}=0$, the instruction command code register is selected, allowing the user to send a command such as clear display, cursor at home, etc. IF $\mathrm{RS}=1$ the data register is selected, allowing the user to send data to be displayed on the LCD.

\section{3 (a) (iii) $R / W$ Read/Write}

$\mathrm{R} / \mathrm{W}$ input allows the user to write information to the LCD or read information from it.

$\mathrm{R} / \mathrm{W}=1$ when reading; $\mathrm{R} / \mathrm{W}=0$ when writing.

\section{3 (a) (iv) E, ENABLE}

The enable pin is used by the LCD to latch information present to its data pins. When data is supplied to data pins, a high to low pulse must be applied to this pin in order for the LCD to latch in 
www.rspsciencehub.com

the data present at the data pins. This pulse must be a minimum of 450 nswide.

\section{$6.3(a)(v) \mathrm{D} 0-\mathrm{D} 7$}

The 8-bit data pins, D0-D7, are used to send information to LCD or read the contents of the LCD's internal registers. The LCD commands codes are as shown in

Table.1. To display letters and numbers, we send ASCII codes for the letters $A-Z, a-z$, and numbers $0-9$ to these pins while making $R S=1$.

\begin{tabular}{|l|l|}
\hline 1 & Clear display screen \\
\hline 2 & Return home \\
\hline 4 & Decrement cursor \\
\hline 6 & Increment cursor \\
\hline 5 & Shift display right \\
\hline 7 & Shift display left \\
\hline 8 & Display off, cursor off \\
\hline A & Display off, cursor on \\
\hline C & Display on, cursor off \\
\hline E & Display on, cursor blinking \\
\hline F & Display on, cursor blinking \\
\hline 10 & Shift cursor position to left \\
\hline 14 & Shift cursor position to right \\
\hline 18 & Shift the entire display to the left \\
\hline 1 C & Shift the entire display to the right \\
\hline 80 & Force cursor to beginning of the $1^{\text {st line }}$ \\
\hline C0 & Force cursor to beginning of the $2^{\text {nd }}$ line \\
\hline 38 & 2 lines and $2 * 7$ matrix \\
\hline
\end{tabular}

\section{3 (b) Speaker:}

Speakers sold for use with computers, although usually capable of other audio uses, e.g. for an MP3 players. Most such speakers have an internal amplifier and consequently require a power source, which may be by a mains power supply often via an AC adapter, batteries, or a USB port. The signal input connector is often a $3.5 \mathrm{~mm}$ jack plug (usually color-coded lime green per the PC 99 standard); RCA connectors are sometimes used, and a USB port may supply both signal and power (requiring additional circuitry, and only suitable for use with a computer). Battery-powered wireless Bluetooth speakers require no connections at all. Computer speakers range widely in quality and in price. Computer speakers sometimes packaged with computer systems are small, plastic, and have mediocre sound quality. Some computer speakers have equalization features such as bass and treble
Volume 02 Issue 04 April 2020

controls. Bluetooth speakers can be connected with a computer by using an Aux jack and compatible adaptor.

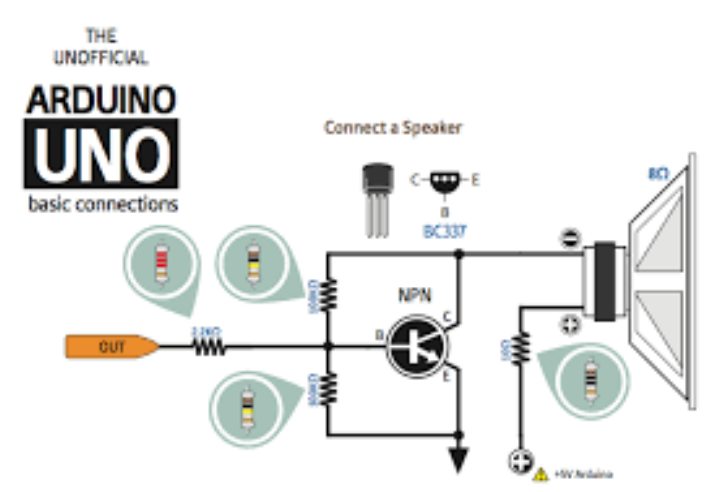

Fig. 16 Circuit diagram of Speaker

More sophisticated computer speakers can have a subwooferunit, to enhance bass output. The larger subwoofer enclosure usually contains the amplifiers for the subwoofer and the left and right speakers.Some computer displays have rather basic speakers built-in. Laptop computers have built-in integrated speakers, usually small and of restricted sound quality to conserve space.

\section{Software Requirements}

\subsection{Embedded C:}

Embedded $\mathrm{C}$ is a lot of language augmentations for the $C$ programming language by the $C$ Standards Committee to address shared characteristic issues that exist between $\mathrm{C}$ expansions for various installed frameworks.

Embedded $\mathrm{C}$ programming normally requires nonstandard augmentations to the $\mathrm{C}$ language so as to help improved chip highlights, for example, fixed-point number juggling, numerous particular memory banks, and fundamental I/O activities. In 2008, the C Standards Committee stretched out the $\mathrm{C}$ language to address such capacities by giving a typical standard to all usage to hold fast to. It incorporates various highlights not accessible in typical $\mathrm{C}$, for example, fixed-point number juggling, named address spaces and fundamental I/O equipment tending to. Inserted $\mathrm{C}$ utilizes the greater part of the language structure and semantics of standard C, e.g., primary () work, variable definition, datatype announcement, contingent articulations (if, switch case), circles (while, for), capacities, clusters and strings, structures and association, bit tasks, macros, and so forth. 


\section{RESULT}

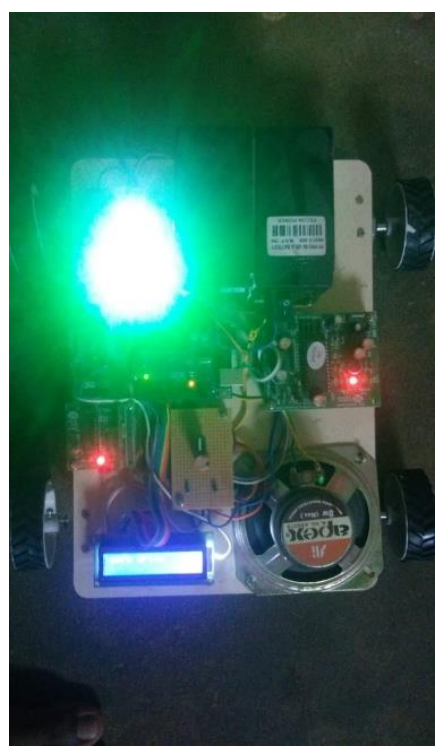

Figure.17. Result

\section{CONCLUSION}

We had introduced the fishermen border alert system controlled by ATMEGA microcontroller i.e. ATMEGA 328. It is a 40 pin IC which having the property of burning a program while running

another program. It is reliable, flexible and of low cost. The alert system which we have developed will provide an effective solution for fishermen's problem and prevent them from crossing other country border. The application can save the lives of many fishermen. The application works as an automatic incident management application that intimates the user if border crossing occurs. If the sailor crosses the border the alarm is used for identification of

fisherman and alert message navy control room and fishermen family. When fishermen reach the sub-border, boat speed will automatically reduced and OTP is given to the navy control room.

\section{Future Scope}

It is proposed as a low cost optimized technique using RFID and GSM mobile technology. At the national border, there will be RFID checking which reduces the threat of terrorism. At the same time, it will generate:

1. Automatic alarmingsystem.
2. Embedded System can design for easy to secure fishermen life using valid RF receiver transmitter

\section{References}

1. J Charles Finny Joseph, R Dinesh Kumar, $M$ Shubin Aldo, "Alert System for Fishermen Crossing Border using Android", International Conference on Electrical, Electronics and optimization Techniques (ICEEOT)-2016.

2. Archana Gupta, Mohammed Abdul Qadeer, Sandeep Kumar Location Based Services using Android, 978-1-4244-47930/09, 2015IEEE.

3. Majid A. Al-Taee, Nabeel A. Al-Saber, Omar B. Khader, "Remote Monitoring of Vehicle Diagnostics and Location Using a Smart Box with Global Positioning System and General Packet Radio Service", 14244- 1031-2 /07, 2018 IEEE. Asian Journal of Applied Science and Technology (AJAST) Volume 1, Issue 1, Pages - 37, February2017.

4. Pulathisi Bandara, Udana Bandara, "Tagciti: A Practical Approach for Location-Aware and Socially-Relevant Information Creation and Discovery for Mobile Users", 978-1-4244-2489-4/08, 2015 IEEE.

5. Patricia Dockhorn Costa, Richard Etter, Tom Broens, "A Rule-Based Approach towards Context-Aware User Notification Services", 1-4244-0237-9/06, 2017.

6. Palanivel Kodeswaran, Vikrant Nandakumar, Shalini Kapoor, Pavan Kamaraju, Anupam Joshi, Sougata Mukherjea, "Securing Enterprise Data on Smartphones using Run Time Information Flow Control", IEEE2016.

7. Sokol Kosta, Andrius Aucinas, Pan Hui, Richard Mortier, Xinwen Zhang, "ThinkAir: Dynamic resource allocation and parallel execution in the cloud for mobile code offloading", IEEE2018.

8. Khandaker Mustakimur Rahman, T.Alam, M.Chowdhury, "Location based early disaster warning and evacuation system on mobile phones using Open Street Map", IEEE2016. 
9. Archana Gupta, Mohammed Abdul Qadeer, Sandeep Kumar LocationBased Services using Android, 978-1-4244-47930/09, 2009IEEE.

10. J Charles Finny Joseph, R Dinesh Kumar, M Shubin Aldo, "AlertSystem for Fishermen Crossing Border using Android", International Conference on Electrical, Electronics and optimization Techniques(ICEEOT)-2016.

11. Khandaker Mustakimur Rahman, T.Alam, M.Chowdhury, "Locationbased early disaster warning and evacuation system on mobile phones using Open Street Map", IEEE2016.

12. Majid A. Al-Taee, Nabeel A. Al-Saber, Omar B. Khader, "Remote Monitoring of Vehicle Diagnostics and Location Using a Smart Box with Global Positioning System and General Packet Radio Service", 14244- 1031-2 /07, 2007 IEEE.

13. Palanivel Kodeswaran, Vikrant Nandakumar, Shalini Kapoor, Pavan Kamaraju, Anupam Joshi, Sougata Mukherjea, "Securing Enterprise Dataon Smartphones using Run Time Information Flow Control", IEEE2016. 